\title{
UNA APROXIMACIÓN DE NÚMEROS DIFUSOS DE TIPO TRAPEZOIDAL
}

\section{AN APPROXIMATION OF TRAPEZOIDAL TYPE FUZZY NUMBERS}

\author{
Y. Chalco-Cano $^{1} \quad$ J. Pedraza Arpasi ${ }^{2} \quad$ H. Román-Flores ${ }^{3} \quad$ G. Álvarez-Jáuregui ${ }^{4}$ \\ Recibido 10 de abril de 2007, aceptado 28 de abril de 2009 \\ Received: April 10, 2007 Accepted: April 28, 2009
}

\begin{abstract}
RESUMEN
En este trabajo se presenta una aproximación para un número difuso de tipo trapezoidal por una secuencia de números difusos $\varepsilon$-diferenciables, es decir, números difusos cuya función de pertenencia es diferenciable en el interior del nivel $\varepsilon$. El proceso consiste en la construcción de un número difuso diferenciable usando la sup-min-convolución de un número difuso con un número difuso simétrico casi Gaussiano. Se presentan algunos ejemplos numéricos y un algoritmo computacional para este proceso.

Palabras clave: Número difuso, número difuso tipo trapezoidal, aproximación de números difusos.

ABSTRACT

In this paper presents an approximation for a trapezoidal type fuzzy number by a sequence of $\varepsilon$-differentiable fuzzy numbers, i.e. fuzzy numbers with differentiable membership function in the interior of the $\varepsilon$-level. The process consists on the construction of a differentiable fuzzy number using sup-min-convolution of a fuzzy number with a quasi-Gaussian fuzzy number. This work shows some numerical examples and a computational algorithm for this process.
\end{abstract}

Keywords: Fuzzy number, trapezoidal type fuzzy number, approximation of fuzzy numbers.

\section{INTRODUCCIÓN}

La teoría de conjuntos difusos es una herramienta importante para modelar incertezas y para procesar información vaga o subjetiva en modelos matemáticos. Sus direcciones de desarrollo han sido diversas y con aplicaciones en diversos problemas reales, por ejemplo, la lógica difusa tiene una gran variedad de aplicaciones, las cuales van desde control de complejos procesos industriales, hasta el diseño de dispositivos artificiales automáticos, pasando por la construcción de artefactos electrónicos de uso doméstico y de entretenimiento, así como también de sistemas de diagnóstico. Una referencia básica sobre conjuntos difusos, lógica difusa y aplicaciones son los libros [2021]. También, podemos encontrar conjuntos difusos en modelos matemáticos aplicados a medicina [2], [3], en sistemas caóticos [9, 22-23], en diversos problemas de ingeniería [15-18, 27], en biología [11-12], etc.
Formulaciones típicas encontradas en la literatura utilizan, para procesar información vaga, conjuntos difusos cuyas funciones de pertenencia son lineales o lineales por tramos, por ejemplo en el diseño electromagnético difuso [27], en optimización difusa [7]. También, es usado en aplicaciones de control difuso donde los recursos computacionales son limitados y no permite el uso de funciones de pertenencia complicadas [15]. Sin embargo, el uso de funciones de pertenencia diferenciables es necesario en diversos casos, tal como ocurre en la implementación de sistemas expertos difusos y sus aplicaciones. Por ejemplo, en modelos de aprendizaje neuro difuso, los cuales están basados en el método del gradiente descendente, es necesario que la función de pertenencia sea diferenciable, es decir, la entrada (input) tiene que ser un conjunto difuso cuya función de pertenencia es diferenciable (ver [4]). También, en otras áreas, los parámetros de los modelos deben ser conjuntos

1 Universidad de Tarapacá. Departamento de Matemática. Casilla 7-D. Arica, Chile. E-mail: ychalco@uta.cl

2 Universidade Regional Integrada Frederico Westphalen. Departamento de Engenharias e Ciência da Computação. Rio Grande do Sul, Brasil

3 Universidad de Tarapacá. Instituto de Alta Investigación. Casilla 7-D. Arica, Chile. E-mail: hroman@uta.cl

4 Universidad Nacional de San Antonio Abad. Facultad de Ciencias. Cusco, Perú. 
difusos cuya función de pertenencia es diferenciable, por ejemplo, en los métodos de optimización difusa que están basados en la gradiente, ver [15]. En particular en [15] se sugiere el uso de un número difuso simétrico casi Gaussiano. Por otro lado, en [13] se resalta que la construcción de funciones de pertenencia diferenciables juega un rol relevante en el modelamiento y resolución de problemas reales.

En [1] se utilizan funciones de pertenencia de tipo casi Gaussiana como funciones de pertenencia diferenciables (ver también [18]). Estas, también son usadas en [17] para el estudio de la influencia de variabilidad difusa en la estimación de conductividad hidráulica y en [16] para problemas de ingeniería mecánica, entre otras aplicaciones.

Ahora, en la teoría de conjuntos difusos existen muchas situaciones donde es necesaria la aproximación de un número difuso por números difusos con propiedades más convenientes. Por ejemplo, en [24] los autores presentan una aproximación de un número difuso por números difusos Lipschitz (función de pertenencia Lipschitz); en [8] se presenta una aproximación de un número difuso por números difusos de tipo trapezoidal; en [5] se muestra una aproximación de un número difuso por números difusos diferenciables casi en todas partes. En esta última aproximación se usa la convolución de un número difuso con un número difuso cuadrático. Otros tipos de aproximación de números difusos es estudiado en los trabajos $[6,10,19,26]$.

En este trabajo se presenta una nueva forma de construir conjuntos difusos diferenciables y, junto con ello, se muestra un nuevo tipo de aproximación para conjuntos difusos de tipo trapezoidal, cuyo proceso es diferente de los existentes. Para esto se usa la sup-min-convolución de un número difuso tipo trapezoidal $u$ con los números difusos simétricos casi Gaussianos $\eta_{n}$, generando la secuencia de números difusos $w_{n}$. Se muestra que $w_{n}$ es un número difuso $\varepsilon$-diferenciable, para cada $n \in \mathrm{N}$ y $\varepsilon \cong 0.011$, los cuales convergen (en $d_{\infty}$-métrica) rápidamente a $u$. Este resultado generaliza la aproximación presentada en [8]. Además, se implementa un algoritmo computacional para este proceso.

\section{PRELIMINARES}

Un conjunto difuso $u$ en un conjunto universo $X$ se representa por una aplicación $u: X \rightarrow[0,1]$, siendo $u(\cdot)$ la función de pertenencia asociada al conjunto difuso $u$. En este caso, la interpretación es que para cada $x \in X$, $u(x)$ representa el grado de pertenencia de $x$ al conjunto difuso $u$.

Para un conjunto difuso $u$ en $\Re$, el conjunto de los números reales, definimos el $\alpha$-nivel de $u$ por $[u]^{\alpha}=\{x \in \Re / u(x) \geq \alpha\}$, con $0<\alpha \leq 1$. Para $\alpha=0$ tenemos el soporte de $u$, el cual es dado por $\operatorname{Supp}(u)=\overline{\{x \in \Re / u(x)>0\}}$, donde $\bar{A}$ denota la cerradura del conjunto $A$. Note que $[u]^{0}$ indica el grado de incerteza del conjunto difuso $u$ (este también es llamado intervalo de incerteza).

Denotamos por $\mathfrak{I}$ la familia de todos los números difusos, es decir, la familia de todo conjunto difuso $u$ en $\Re$ tal que $[u]^{\alpha}$ es un intervalo cerrado y limitado para cada $\alpha$ $\in[0,1]$.

Para cualquier número difuso $u$ existen cuatro (4) números reales $a, b, c, d$ y dos funciones semicontinuas superior $L:[a, b] \rightarrow[0,1]$ y $R:[c, d] \rightarrow[0,1]$, donde $L$ es una función no decreciente y $R$ es una función no creciente con $L(b)=R(c)=1$, tal que $u$ puede ser descrito de la siguiente manera

$$
u(x)=\left\{\begin{array}{ccc}
0 & \text { si } & x<a \\
L(x) & \text { si } & a \leq x<b \\
1 & \text { si } & b \leq x \leq c \\
R(x) & \text { si } & c<x \leq d \\
0 & \text { si } & x>d .
\end{array}\right.
$$

Denotamos por $\mathfrak{I}_{T}$ la familia de todos los números difusos de tipo trapezoidal, es decir, la familia de todo número difuso $u$ tal que $L$ es estrictamente creciente en $[a, b]$, diferenciable en $(a, b)$ y $R$ es estrictamente decreciente en $[c, d]$, diferenciable en $(c, d)$ y $L(a)=R(d)=0$. Es claro que esta familia $\mathfrak{I}_{T}$ contiene a los números difusos triangulares.

Si $u \in \mathfrak{I}_{T}$ entonces tenemos que

$$
[u]^{\alpha}=\left[L^{-1}(\alpha), R^{-1}(\alpha)\right],
$$

para cada $\alpha \in[0,1]$, donde $L^{-1}$ y $R^{-1}$ son las funciones inversas de $L$ y $R$, respectivamente.

También, denotemos por $\mathfrak{I}_{D(\varepsilon)}$ la familia de todos los números difusos $\varepsilon$-diferenciables, es decir, la familia de todos los números difusos $u$ tal que $u$ es diferenciable en el interior de $[u]^{\varepsilon}$. 
De las definiciones anteriores, notemos que $\mathfrak{I}_{T} \subset \mathfrak{I}$. Esta inclusión es estricta, como vemos en las figuras 1 y 2 , donde $w \in \mathfrak{I}$ pero $w \notin \mathfrak{I}_{\mathrm{T}}$.

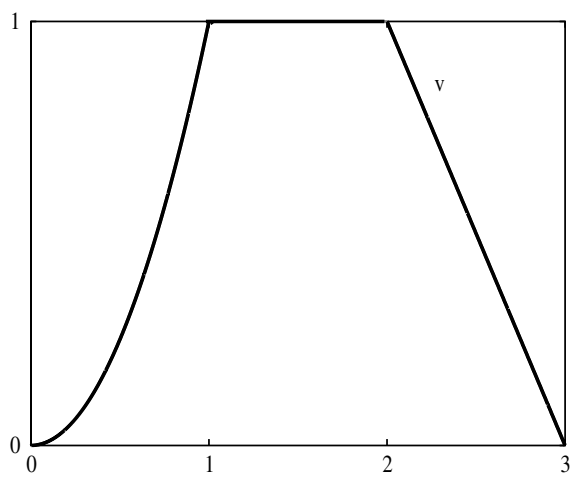

Figura 1. Número difuso tipo trapezoidal.

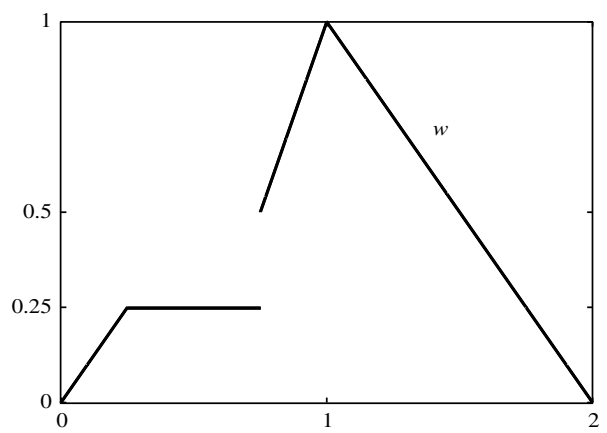

Figura 2. Número difuso que no es tipo trapezoidal.

Un número difuso simétrico casi Gaussiano es definido por (ver [17-18])

$$
\eta(x)=e^{-\frac{(x-\bar{m})^{2}}{2 \sigma^{2}}}
$$

para $|x=\bar{m}| \leq 3 \sigma$ y $\eta(x)=0$ para $x>\bar{m}+3 \sigma$ o $x<\bar{m}-3 \sigma$, donde $\bar{m}$ y $\sigma$ denotan el valor medio y la desviación estándar de la distribución Gaussiana, respectivamente. La figura 3 es un número difuso casi Gaussiano, con valor medio 0 y desviación estándar 1/3.

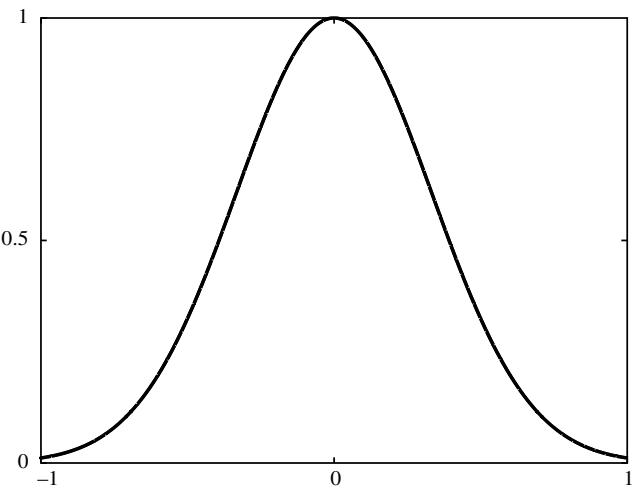

Figura 3. Número difuso casi Gaussiano, con valor medio 0 y desviación estándar 1/3.

Observación 1.- El número difuso simétrico casi Gaussiano fue motivo de estudio de varios autores y usado en los más diversos tópicos, ver [1, 15, 17-18] y referencias. Cabe resaltar que este número difuso es un número difuso $\varepsilon$-diferenciable, con $\varepsilon \cong 0.011$, ya que tiene la propiedad de ser diferenciable en el interior $[\eta]^{\alpha}=[\eta]^{0}$, no es diferenciable en los extremos del mismo y no es un número difuso tipo trapezoidal según la definición de $\mathfrak{I}_{\mathrm{T}}$, ver figura 3. Esto quiere decir que el menor grado de pertenencia para los elementos del soporte de $\eta$ es $\mathcal{E}$ y este es bastante "pequeño". Pero, por las propiedades interesantes de la distribución Gaussiana en la teoría de probabilidades, hace que este número difuso sea importante en la teoría de conjuntos difusos, ver [1, 15, 17-18].

Las operaciones de suma y multiplicación por un escalar en el espacio $\mathfrak{I}$ son generadas por el principio de extensión (véase [20]). Pero, a su vez, estas operaciones son equivalentes a:

$$
(u+v)(x)=\sup _{y+z=x}\{u(y) \wedge v(z)\}
$$

$\mathrm{y}$

$$
(\lambda \cdot u)(x)=\left\{\begin{array}{ccc}
u\left(\frac{x}{\lambda}\right) & \text { si } & \lambda \neq 0 \\
\chi_{\{0\}}(x) & \text { si } & \lambda=0 .
\end{array}\right.
$$

donde $\chi_{\{0\}}$ es la función característica de $\{0\}$ y $\wedge$ denota el mínimo entre dos números.

También, para cada $\alpha \in[0,1]$ las siguientes relaciones se cumplen

$$
[u+v]^{\alpha}=[u]^{\alpha}+[v]^{\alpha} \text { y }[\lambda \cdot u]^{\alpha}=\lambda \cdot[u]^{\alpha} .
$$


Note que la suma de dos números difusos $u$ y $v$ dada en (1) es equivalente a la sup-min-convolución $u \nabla v$, tópico bastante conocido en la teoría de análisis convexo [25]. Más precisamente,

$$
(u \nabla v)(x)=(u+v)(x)=\sup _{y \in \mathfrak{R}}\{u(y) \wedge v(x-y)\} .
$$

Esta definición fue estudiada y usada por los autores en [24] para la aproximación de un conjunto difuso por una secuencia de conjuntos difusos Lipschitz. También, en [5] fue estudiada la convolución de un número difuso con un número difuso cuadrático, generando un número difuso casi diferenciable.

Para dos números difusos $u$ y $v$ cualesquiera, la distancia entre ambos es dada por

$$
d_{\infty}(u, v)=\sup _{0 \leq \alpha \leq 1} H\left([u]^{\alpha},[v]^{\alpha}\right),
$$

donde $H$ es la métrica de Hausdorff que es definida por

$$
H([a, b],[c, d])=\max \{|a-c|,|b-d|\},
$$

para cada par de intervalos cerrados y limitados $[a, b]$, $[c, d]$.

Dados $u, v$ y $w$ números difusos, se cumple que

$$
d_{\infty}(u+w, v+w) \leq d_{\infty}(u, v) .
$$

\section{APROXIMACIÓN}

Denotemos por $\eta_{p}$ el número difuso casi Gaussiano con media $\bar{m}=0$ y desviación estándar $\sigma=p / 3$, es decir,

$$
\eta_{p}=\left\{\begin{array}{ccc}
-\frac{9 x^{2}}{2 p^{2}} & \text { si } \quad-p \leq x \leq p \\
0 & \text { si } & x \notin[-p, p] .
\end{array}\right.
$$

Para cada $\alpha \in[0,1]$ tenemos

$$
\left[\eta_{p}\right]^{\alpha}=\left\{\begin{array}{c}
{\left[-\sqrt{-\frac{2 p^{2}}{9} \ln (\alpha),} \sqrt{-\frac{2 p^{2}}{9} \ln (\alpha)}\right] \text { si } e^{-9 / 2} \leq \alpha \leq 1} \\
{[-p, p] \text { si } \quad 0 \leq \alpha \leq e^{-9 / 2} .}
\end{array}\right.
$$

En esta sección tomamos

$$
\varepsilon=e^{-\frac{9}{2}} \cong 0.011
$$

Construiremos una secuencia de números difusos $\varepsilon$-diferenciables $w_{n}=u \nabla \eta_{1 / n}$, a partir de un número difuso $u$. Mostraremos también que la secuencia $w_{n}$ converge a $u$ en la métrica $d_{\infty}$.

Antes de mostrar la convergencia, presentamos una propiedad de regularización asociada a la convolución de un número difuso de tipo trapezoidal $u$ con $\eta_{p}$.

Teorema 1.- Sea $u$ un número difuso. Si $u \in \mathfrak{I}_{T}$ entonces $u \nabla \eta_{p} \in \mathfrak{I}_{D(\varepsilon)}$

Demostración Como $u$ es un número difuso tipo trapezoidal, entonces podemos considerar $u$ como en (1) donde $L$ y $R$ son diferenciables en $(a, b)$ y $(c, d)$, respectivamente, y son estrictamente monótonas. También, tenemos que

$$
[u]^{\alpha}=\left[L^{-1}(\alpha), R^{-1}(\alpha)\right] .
$$

\section{Luego}

$$
\begin{aligned}
& {\left[u \nabla \eta_{p}\right]^{\alpha}=} \\
& \left\{\left[\left[L^{-1}(\alpha)-\sqrt{\left.-\frac{2 p^{2}}{9} \ln (\alpha), R^{-1}(\alpha)+\sqrt{-\frac{2 p^{2}}{9} \ln (\alpha)}\right] \text { si } e^{-\frac{9}{2}} \leq \alpha \leq 1}\right.\right.\right. \\
& {\left[L^{-1}(\alpha)-p, R^{-1}(\alpha)+p\right] \text { si } 0 \leq \alpha \leq e^{-\frac{9}{2}} .}
\end{aligned}
$$

Ahora, consideremos

$$
f(\alpha)=\left\{\begin{array}{c}
L^{-1}(\alpha)-\sqrt{-\frac{2 p^{2}}{9} \ln (\alpha)} \quad \text { si } e^{-\frac{9}{2}} \leq \alpha \leq 1 \\
L^{-1}(\alpha)-p \text { si } 0 \leq \alpha \leq e^{-\frac{9}{2}}
\end{array}\right.
$$

y

$$
g(\alpha)=\left\{\begin{array}{c}
R^{-1}(\alpha)+\sqrt{-\frac{2 p^{2}}{9} \ln (\alpha)} \text { si } e^{-\frac{9}{2}} \leq \alpha \leq 1 \\
R^{-1}(\alpha)+p \text { si } 0 \leq \alpha \leq e^{-\frac{9}{2}}
\end{array}\right.
$$


entonces $f$ y $g$ son funciones biyectivas en $[0,1]$ y diferenciables en $(\varepsilon, 1) y$, además, se tiene que

$$
\left(u \nabla \eta_{p}\right)(x)=\left\{\begin{array}{lll}
0 & \text { si } & x<a-p \\
f^{-1}(x) & \text { si } & a-p \leq x \leq b \\
1 & \text { si } & b \leq x \leq c \\
g^{-1}(x) & \text { si } & c \leq x \leq d+p \\
0 & \text { si } & d+p<x
\end{array}\right.
$$

Luego, $u \nabla \eta_{p}=f^{-1}$ es diferenciable en $\left(L^{-1}(\varepsilon)-p, b\right)$ y $u \nabla \eta_{p}=g^{-1}$ es diferenciable en $\left(c, R^{-1}(\varepsilon)+p, b\right)$. Para completar la demostración, mostramos que

$$
\frac{d\left(u \nabla \eta_{p}\right)}{d x}(b)=\frac{d\left(u \nabla \eta_{p}\right)}{d x}(c)=0
$$

En efecto,

$$
\lim _{x \rightarrow b^{+}} \frac{\left(u \nabla \eta_{p}\right)(x)-\left(u \nabla \eta_{p}\right)(b)}{x-b}=0 .
$$

Considerando $f^{-1}(x)=\alpha$ y tomando en cuenta la regla de L'Hopital,

$$
\begin{aligned}
& \lim _{x \rightarrow b^{-}} \frac{\left(u \nabla \eta_{p}\right)(x)-\left(u \nabla \eta_{p}\right)(b)}{x-b} \\
= & \lim _{x \rightarrow b^{-}} \frac{f^{-1}(x)-f^{-1}(b)}{x-b} \\
= & \lim _{\alpha \rightarrow 1^{-}} \frac{\alpha-1}{L^{-1}(\alpha)-\sqrt{-\frac{2 p^{2}}{9} \ln (\alpha)-b}} \\
= & \lim _{\alpha \rightarrow 1^{-}} \frac{2 \alpha \sqrt{-\ln (\alpha)}}{2 \alpha \sqrt{-\ln (\alpha)}\left(L^{-1}\right)^{\prime}(\alpha)+\sqrt{\frac{2 p^{2}}{9}}}=0 .
\end{aligned}
$$

Por lo tanto, $\frac{d\left(u \nabla \eta_{p}\right)}{d x}(b)=0$.

Similarmente $\frac{d\left(u \nabla \eta_{p}\right)}{d x}(c)=0$. Así, $u \nabla \eta_{p} \in \mathfrak{I}_{D(\varepsilon)}$.

Teorema 2.- Sea $u$ un número difuso. Si $u \in \mathfrak{I}_{T}$ entonces existe una secuencia de números difusos $\left(w_{n}\right) \subset \mathfrak{I}_{\mathrm{D}(\varepsilon)}$ tal que

$$
d_{\infty}\left(w_{n}, u\right)<1 / \mathrm{n},
$$

para todo $n=1,2,3, \ldots$

Demostración: Sea $u$ número difuso tal que $u \in \mathfrak{I}_{T}$. Si tomamos $w_{n}=u \nabla \eta_{1 / n}$ entonces, por el Teorema 1, tenemos que $w_{n}$ es un número difuso $\varepsilon$-diferenciable, para $n=1,2$, $3, \ldots$. Además, de la propiedad (3) de la métrica $d_{\infty}$,

$$
\begin{gathered}
d_{\infty}\left(w_{n}, u\right)=d_{\infty}\left(u \nabla \eta_{1 / n}, u\right) \\
\leq d_{\infty}\left(\eta_{1 / n}, \chi_{\{0\}}\right)=1 / n .
\end{gathered}
$$

para todo $n=1,2,3, \ldots$

\section{Observación 2.}

a) Del Teorema 2, concluimos que un número difuso tipo trapezoidal se puede aproximar por una secuencia de números difusos $\varepsilon$-diferenciables, para $\varepsilon \cong 0.011$. Destacamos que la convergencia es dada en la métrica $d_{\infty}$, la cual es una métrica fuerte en la teoría de conjuntos difusos.

b) Note que el número difuso casi Gaussiano $\eta$ y los números difusos aproximantes $w_{n}$ tienen similar comportamiento de diferenciabilidad, en el sentido que son diferenciables en el interior del nivel $\varepsilon$ y no son diferenciables en los extremos del nivel $\varepsilon$.

\section{UN ALGORITMO SIMPLE}

Proponemos un algoritmo computacional que nos permite visualizar varias aproximaciones $w_{n}$. Este algoritmo es el siguiente:

\section{APROXIMACION-DIFUSO (n,L,R) Return $w_{n}$} input: $\mathrm{n}$ número de aproximaciones

L función creciente (lado izquierdo) de $\boldsymbol{u}$ $\mathrm{R}$ función decreciente (lado derecho) de $\boldsymbol{u}$ $\mathrm{L}_{\eta}$ función creciente (lado izquierdo) de $\eta_{1 / n}$ $\mathrm{R}_{\eta}$ función decreciente (lado derecho) de $\eta_{1 / n}$ 
$\mathrm{Li}=\operatorname{INVERSE}(\mathrm{L}(\alpha))$

$\mathrm{Ri}=\operatorname{INVERSE}(\mathrm{R}(\alpha))$

$\mathrm{f}=\mathrm{Li}-\mathrm{L}_{\eta}$

$\mathrm{g}=\mathrm{Ri}+\mathrm{R}_{\eta}$

fn=INVERSE(f)

gn=INVERSE $(\mathrm{g})$

return: fn y gn

Ejemplo 1.- Consideremos el número difuso de tipo trapezoidal $u$ (figura 4) dado por

$$
u(x)=\left\{\begin{array}{ccc}
x^{2} & \text { si } & 0 \leq x \leq 1 \\
1 & \text { si } & 1 \leq x \leq 2 \\
-x+3 & \text { si } & 2 \leq x \leq 3
\end{array}\right.
$$

Este conjunto difuso no es diferenciable en $x=1$ y en $x=2$. Por otro lado, para cada $\alpha \in[0,1]$, sus niveles son dados por

$$
[u]^{\alpha}=[\sqrt{\alpha}, 3-\alpha]
$$

Usando el algoritmo propuesto tenemos los gráficos de los números difusos $\boldsymbol{\varepsilon}$-diferenciables $w_{1}, w_{2}, w_{3} \mathrm{y} w_{4}$ que se aproximan a $u$, como se muestra en la figura 5 .

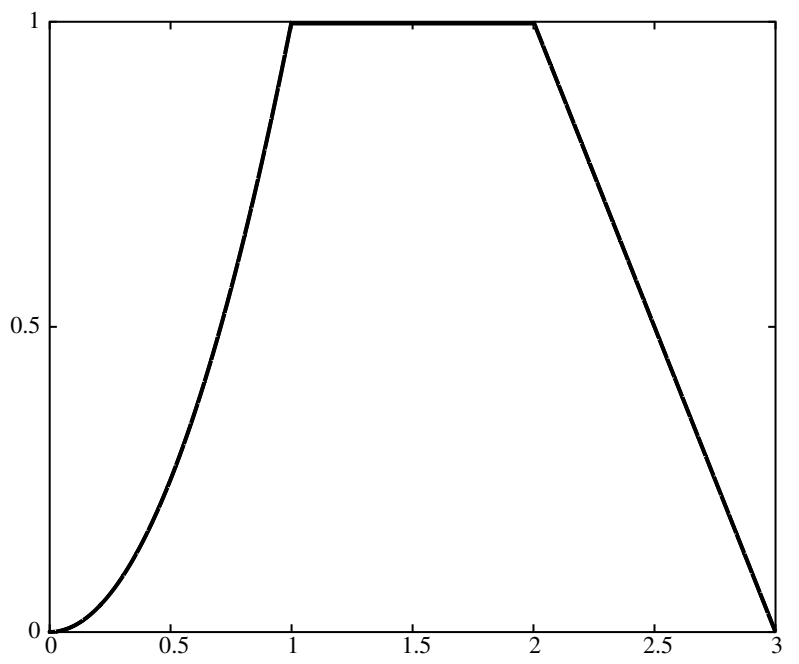

Figura 4. El número difuso $u$.

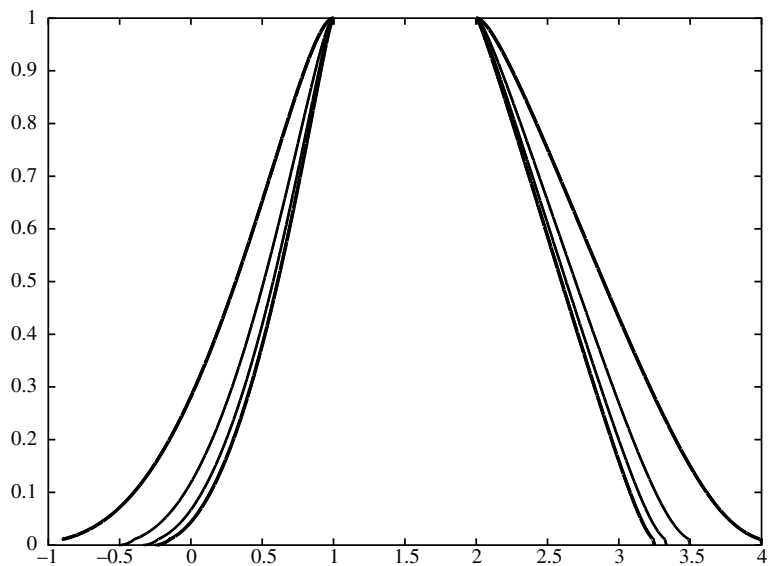

Figura 5. Las aproximaciones $w_{1}, w_{2}, w_{3}$ y $w_{4}$.

Por otro lado, haciendo un zoom al gráfico anterior podemos ver que $w_{1}$ no es diferenciable en los extremos del nivel $\varepsilon$, como podemos ver en la figura 6 .

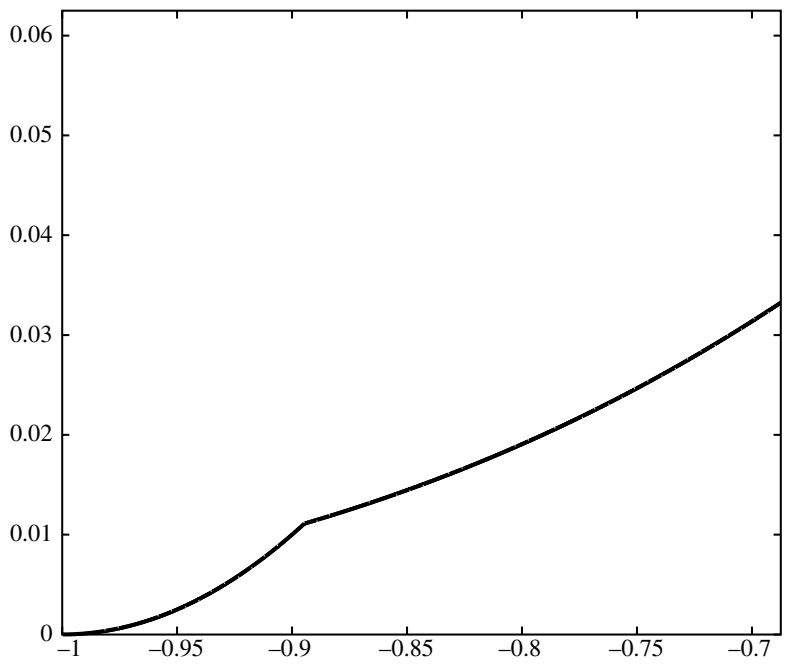

Figura 6. La aproximación $w_{1}$ no es diferenciable en los puntos extremos del nivel $\varepsilon$.

\section{CONCLUSIONES}

En este trabajo hemos presentado una nueva forma de aproximar un número difuso tipo trapezoidal por números difusos $\varepsilon$-diferenciables, con $\varepsilon \cong 0.011$.

La convergencia es dada en la métrica $d_{\infty}$, la cual es una métrica fuerte en la teoría de conjuntos difusos. Esto hace que nuestro algoritmo mejore substancialmente el algoritmo presentado en [8], donde se obtiene una aproximación de un número difuso tipo trapezoidal usando la función sigmoidal, mas los conjuntos difusos 
aproximantes no son números difusos, porque para valores cerca de $\alpha=1$ los niveles son conjuntos vacíos y así no existe convergencia en la métrica $d_{\infty}$. También, cabe resaltar que los números difusos $w_{n}$ tienen propiedades similares de diferenciabilidad con el número difuso casi Gaussiano $\eta$.

Por otro lado, las operaciones matemáticas involucradas en la construcción del algoritmo, la convergencia rápida, así como la implementación computacional del mismo, hacen que el algoritmo sea eficiente.

En teoría difusa, el empleo de funciones de pertenencia diferenciables es con el fin de permitir el uso de métodos basados en gradientes. Por ejemplo, es necesario funciones de pertenencia diferenciables en el diseño de un micromotor electrostático [15]. Así, cada vez que surjan en el modelo funciones de pertenencia trapezoidales, estas necesitan ser aproximadas por funciones de pertenencia diferenciables. En esta instancia el resultado de este trabajo puede ser usado.

\section{AGRADECIMIENTOS}

Este trabajo fue financiado por el proyecto FONDECYT No 1080438 y Ministerio de Ciencia e Innovación, España, proyecto MTM2008-00018.

\section{REFERENCIAS}

[1] P. Alotto, A. Bertoni, G. Molinari, M. Nervi, B. Brandstaetter, Ch. Magele, K.R. Richter, C. Ragusa and M. Repetto. "A combined approach for the stochastic optimization of multiminima problems using adaptive fuzzy sets and radial basis functions". IEEE Transactions on Magnetics. Vol. 34, pp. 2837- 2840. 1998.

[2] M. F. Abbod, D.G. Von Keyserlingk, D.A. Linkens and M. Mahfouf. "Survey of utilisation of fuzzy technology in medicine and healthcare". Fuzzy Sets and Systems. Vol. 120, pp. 331-349. 2001.

[3] S. Barro and R. Marín. "Fuzzy Logic in Medicine". Heidelberg: Physica-Verlag. 2002.

[4] G. Castellano, A. M. Fanelli, C. Mencar. "An empirical risk functional to improve learning in a fuzzy-neuro classifier". IEEE Trans. Syst., Man. Cyber. Vol. 34, pp. 725-731. 2004.
[5] Y. Chalco-Cano, H. Román-Flores and F. Gomide. "A new type of approximation for fuzzy numbers". Fuzzy Sets and Systems. Vol. 159, pp. 1376-1383. 2008.

[6] Y. Chalco-Cano, M. Jiménez-Gamero, H. RománFlores and M.A. Rojas-Medar. "An approximation to the extension principle using decomposition of fuzzy intervals”. Fuzzy Sets and Systems. Vol. 159, pp. 3245-3258. 2008.

[7] H. Cheng-Feng and F. Shu-Cherng. "Solving fuzzy inequalities with piecewise linear membership functions". IEEE Transactions on Fuzzy Systems. Vol. 7, pp. 230-235. 1999.

[8] J. Dombi and Z. Gera. "The approximation of piecewise linear membership functions and Lukasiewicz operators". Fuzzy Sets and Systems Vol. 154, pp. 275-286. 2005.

[9] G. Feng and G. Chen. "Adaptative control of discrete-time chaotic systems: a fuzzy control approach". Chaos Solitons \& Fractals. Vol. 253, pp. 459-467. 2005.

[10] G. González-Rodríguez, A. Colubi and W. Trutschnig. "Simulation of fuzzy random variables". Information Sciences. Vol. 179, pp. 642653. 2009.

[11] M. Guo, X. Xue and R. Li. "The oscillation of delay differential inclusions and fuzzy biodynamics models". Mathematical and Computer Modelling. Vol. 37, pp. 651-658. 2003.

[12] M. Guo and R. Li. "Impulsive functional differential inclusions and fuzzy population models". Fuzzy Sets and Systems. Vol. 138, pp. 601-615. 2003.

[13] A. Grauel and L.A. Ludwig. "Construction of differentiable membership functions". Fuzzy Sets and Systems. Vol. 101, pp. 219-225. 1999.

[14] P. Grzegorzewski and E. Mrówka. "Trapezoidal approximation of fuzzy numbers". Fuzzy Sets and Systems. Vol. 153, pp. 115-135. 2005.

[15] F.G. Guimarães, F. Campelo, R.R. Saldanha and J.A. Ramírez. "A hybrid methodology for fuzzy optimization of electromagnetic devices". IEEE Transactions on Magnetics. Vol. 41, pp. 1744-1747. 2005. 
[16] M. Hanss. "On the implementation of fuzzy arithmetical operations for engineering problems". Proc. of the $18^{\text {th }}$ International Conference of the North American Fuzzy Information Processing Society-NAFIPS '99, pp. 462-466. New York, USA. 1999.

[17] M. Hanss and A.P. Selvadurai. "Influence of fuzzy variability on the estimation of hydraulic conductivity of transversely isotropic geomaterials". Proc. of the NUMOG VIII-International Symposium on Numerical Models in Geomechanics, pp. 675-680. Rome, Italy. 2002.

[18] M. Hanss. "Applied Fuzzy Arithmetic: An Introduction with Engineering Applications". Springer Verlag. Berlin, Alemania. 2005.

[19] E.N. Nasibov and S. Peker. "On the nearest parametric approximation of a fuzzy number". Fuzzy Sets and Systems. Vol. 159, pp. 1365-1375. 2008.

[20] W. Pedricz and F. Gomide. "An Introduction to Fuzzy Sets: Analysis and Design". Massachusetts, USA. MIT Press. 1998.

[21] T.J. Ross. "Fuzzy Logic with Engineering Applications". John Wiley \& Sons Ltd., England. 2005.
[22] H. Román-Flores and Y. Chalco-Cano. "Robinson's chaos in set-valued discrete systems". Chaos Solitons \& Fractals. Vol. 25, pp. 33-42. 2005.

[23] H. Román-Flores and Y. Chalco-Cano. "Some chaotic properties of Zadeh's extension". Chaos Solitons \& Fractals. Vol. 35, pp. 452-459. 2008.

[24] H. Román-Flores, Y. Chalco-Cano and M.A. Rojas-Medar. "Convolution of fuzzy sets and applications". Computers Mathematics with Applications. Vol. 46, pp. 1245-1251. 2003.

[25] T. Rockafellar. "Convex Analysis". Princeton Universy Press. Princeton, New Jersey. 1970.

[26] L. Stefanini, L. Sorini and M.L. Guerra. Parametric representation of fuzzy numbers and application to fuzzy calculus". Fuzzy Sets and Systems Vol. 157, pp. 2423-2455. 2006.

[27] D. Srinivasan, S. Ratnajeevan and H. Hoole. "Fuzzy multiobject optimization for the starting desing of a magnetic circuit". IEEE Transactions on Magnetics. Vol. 32, pp. 1230-1233. 1996. 